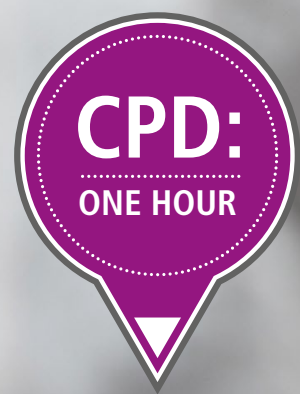

\title{
An introduction to aesthetic dentistry
}

Irfan Ahmad' discusses the seven deadly sins of aesthetic dentistry.

\section{Introduction}

Being in the dental profession, we have a voyeuristic tendency to look at other people's teeth. For example, in socially interactive situations, while chatting at the bus stop, gym, restaurant or wine bar, and non-interactive situations while walking down the street or watching television. On many occasions, aesthetic dental problems are obvious, such as stained or grossly misaligned teeth. However, how many times have you thought that something isn't quite right, but you just can't quite put your finger on it? These less obvious anomalies, especially when maxillary anterior teeth have been restored, make you think that something is wrong. Aesthetic dental restorations (fillings, veneers, crowns

\section{Author information}

'Private Practice, London, UK

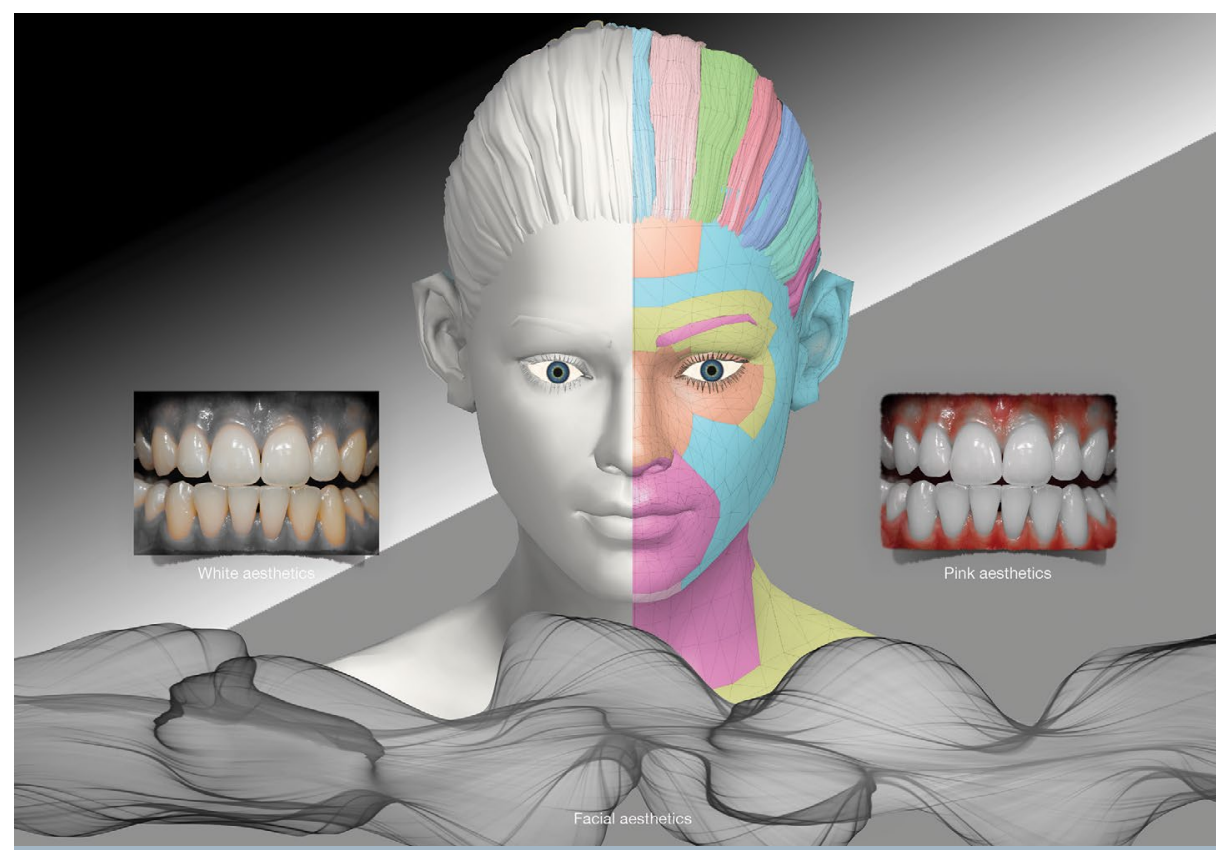

Fig. 1 Dental aesthetics consist of white aesthetics, pink aesthetics and facial aesthetics 


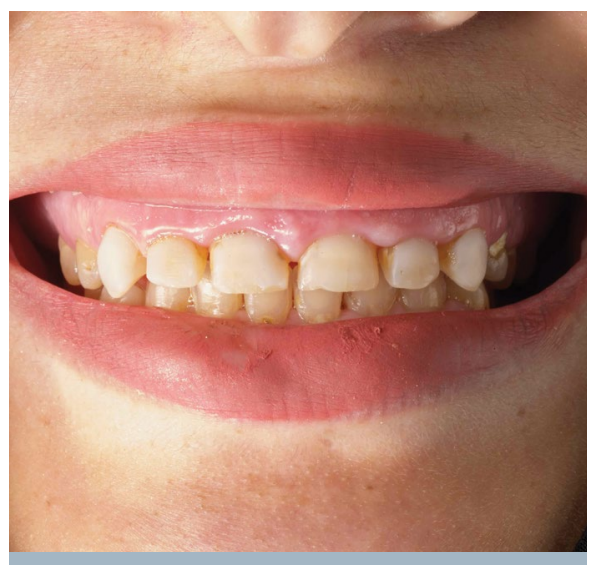

Fig. 2 Digital smile design: pre-operative photograph showing short, wide maxillary anterior teeth

or bridges), if correctly fabricated, should not stick out like a 'sore thumb' but mimic a natural dentition, integrating with the surrounding hard and soft tissues.

\section{What is dental aesthetics?}

Aesthetics is a branch of psychology described as converting a tangible sensation to a psychological (usually pleasurable) experience. This applies to all works of art including literature and the visual arts, whereby a tangible sensation, such as sight and sound, are converted into an intangible psychological experience, eg reading a book, viewing a painting, hearing music, eating a tasty meal or touching someone you love. Dental aesthetics is a broad term concentrating on a smile, and composed of three constituents: white aesthetics (concerning the teeth), pink aesthetics (concerning the gingiva) and facial aesthetics (concerning extra-oral features, predominantly the lips) - Figure 1.

Aesthetics or beauty has preoccupied scientists, scholars and artists for several millennia, who have tried to decipher this elusive phenomenon. The three main theories relating to aesthetics are geometric (mathematical computations for analysing and creating beauty), psychological (relating to an individual's psychoemotional make-up moulded by academic, cultural, social, economic and religious status), and perceptual (social interaction and persona). The basic difference between psychological and perceptual theories is, psychology is the way we 'see' ourselves, while perception is the way others 'see' us. The geometric theories date back to ancient Egyptian, around 3000 $\mathrm{BCE}$, and form the foundation of dental aesthetics. However, most of these concepts have been subsequently discredited for dental use, especially the Golden proportion, which was (and perhaps still is) used to exonerate

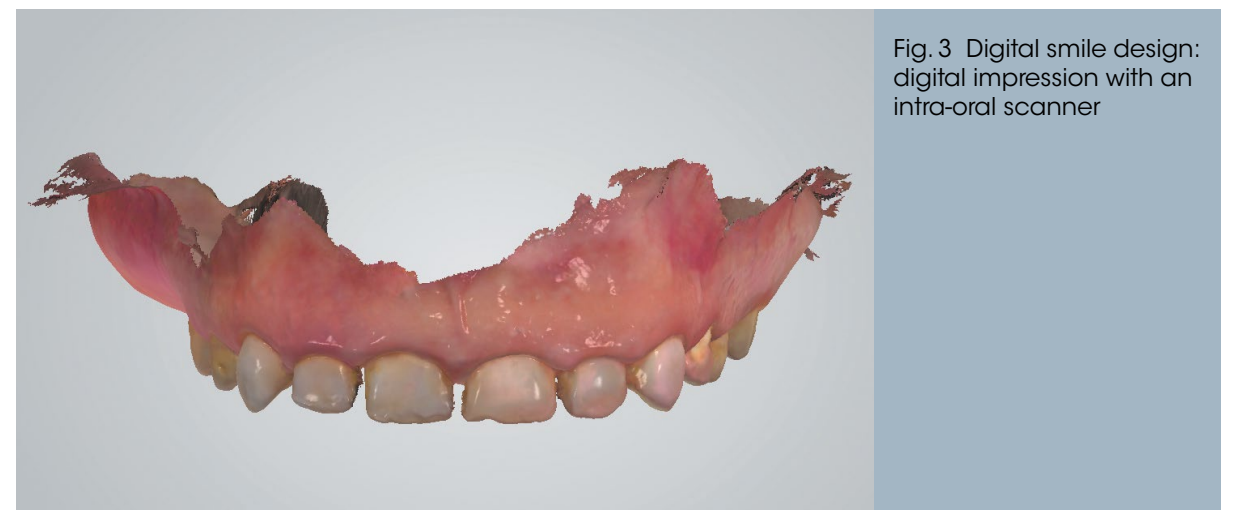

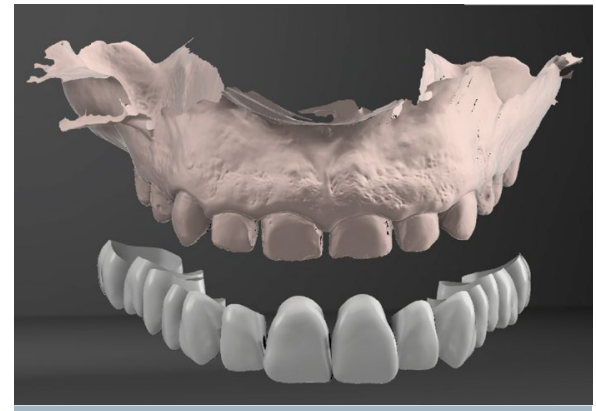

Fig. 4 Digital smile design: CAD (computer aided design) virtual wax-up

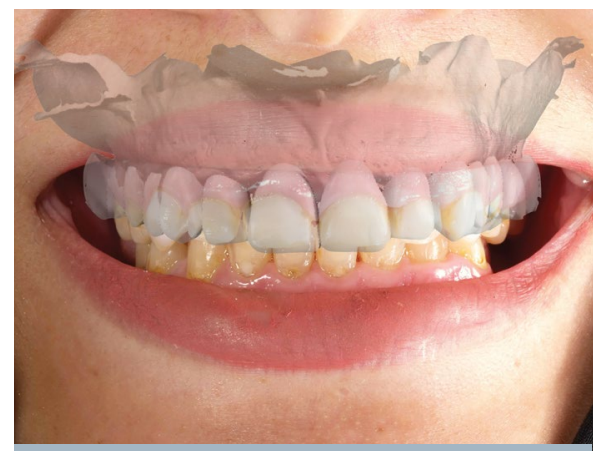

Fig. 6 Digital smile design: virtual wax up merged or 'stitched' with pre-operative photograph to assess white aesthetics (morphology, size, alignment of teeth), pink aesthetics (relation to the surrounding gingiva) and facial aesthetics (relationship to lips)

over-treatment for creating 'perfect smiles' Nevertheless, mathematical concepts are useful as a framework, and are the building blocks of smile designing, but with caveat that there is no magic formula for creating beauty. A comprehensive aesthetic treatment plan incorporates all three theories so that the outcome fulfils the desires of the patient, rather than echoing the opinions or beliefs of the clinician or ceramist.

\section{Aesthetic dentistry}

On a continuum, aesthetic dental treatment ranges from innocuous tooth whitening (bleaching), direct composite fillings, indirect veneers, crowns and bridges to complex implant therapy. Today, we are fortunate to have at our disposal sophisticated restorative
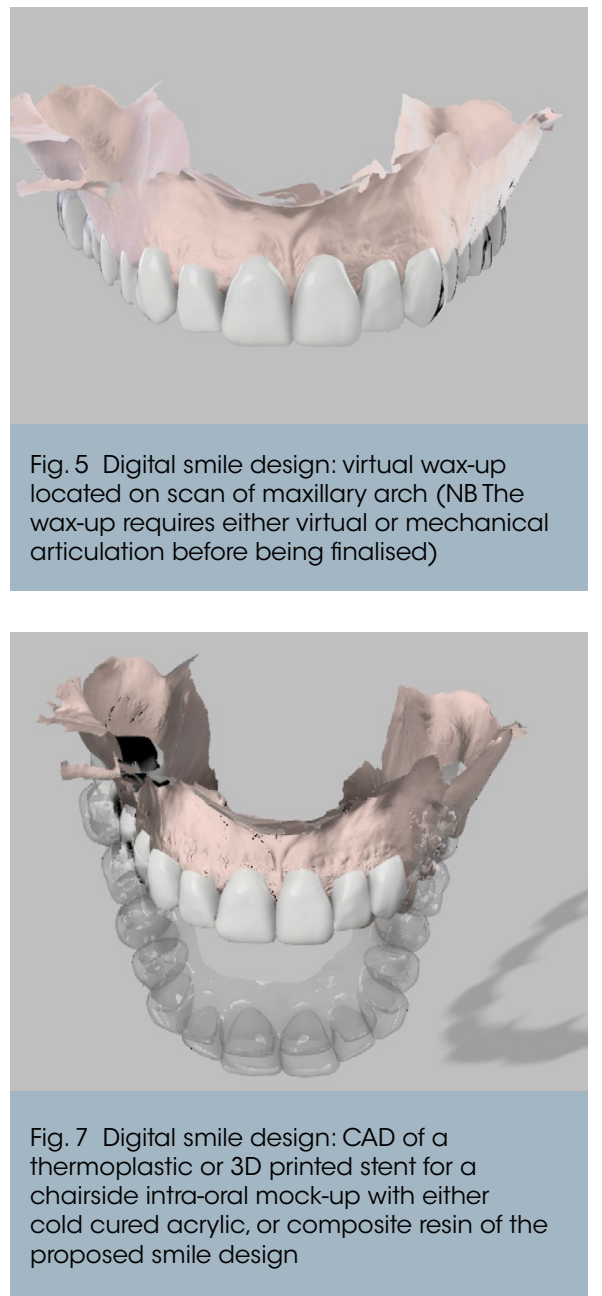

materials, predictable clinical techniques, digital acquisition devices (intra-oral scanners for digital impressions, cone beam computed tomography for 3D visualisation of osseous anatomy, facial scanners for emulating facial expressions), CAD/CAM fabrication, $3 \mathrm{D}$ printing, and innumerable software for diagnosis, treatment planning and digital smile design, Figs 2, 3, 4, 5, 6, 7. Dentistry is currently undergoing a period of renascence.

However, before embarking on an action plan for restituting dental aesthetics, it is worth considering the tenets of evidencebased treatment planning, especially if the contemplated procedures are purely cosmetic rather than analeptic (Fig. 8). As aesthetic 


\section{FEATURE}

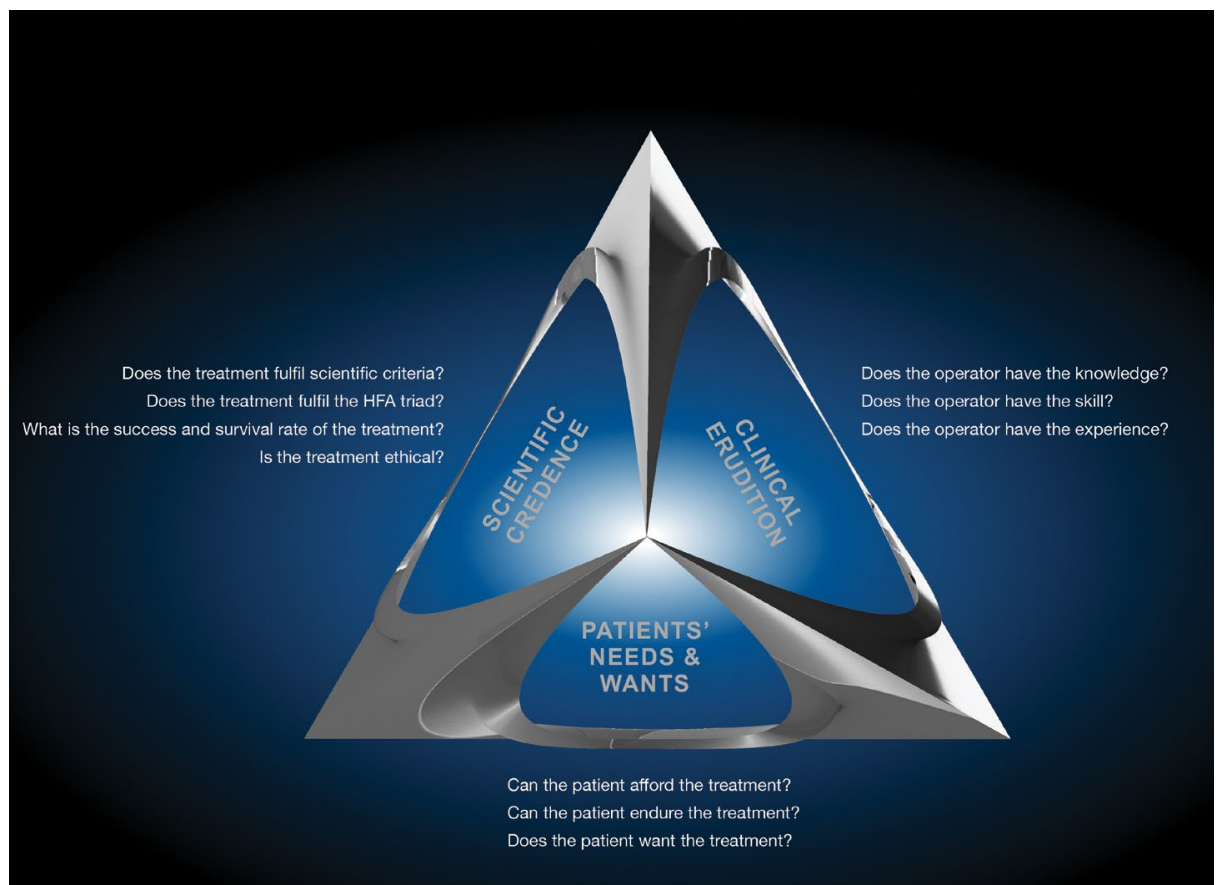

Fig. 8 Evidence-based treatment planning consists of scientific credence, clinical erudition, and patients' needs and wants

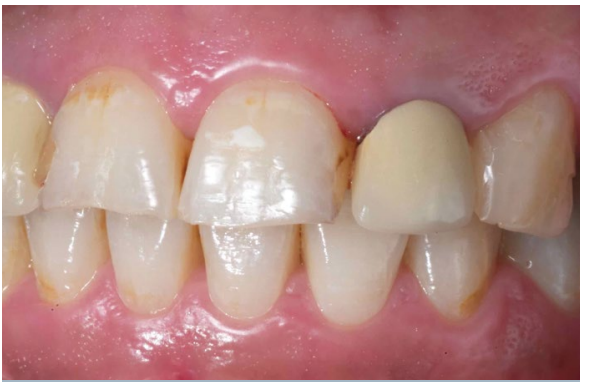

Fig. 10 The shade of the crown on the maxillary left lateral incisor fails to integrate with the surrounding natural teeth

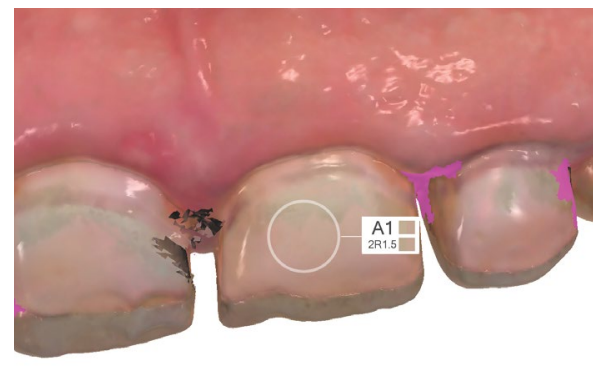

Fig. 11 Intra-oral scanner numerical readout of tooth shade corresponding to VITA classical and VITA 3D-Master shade guides

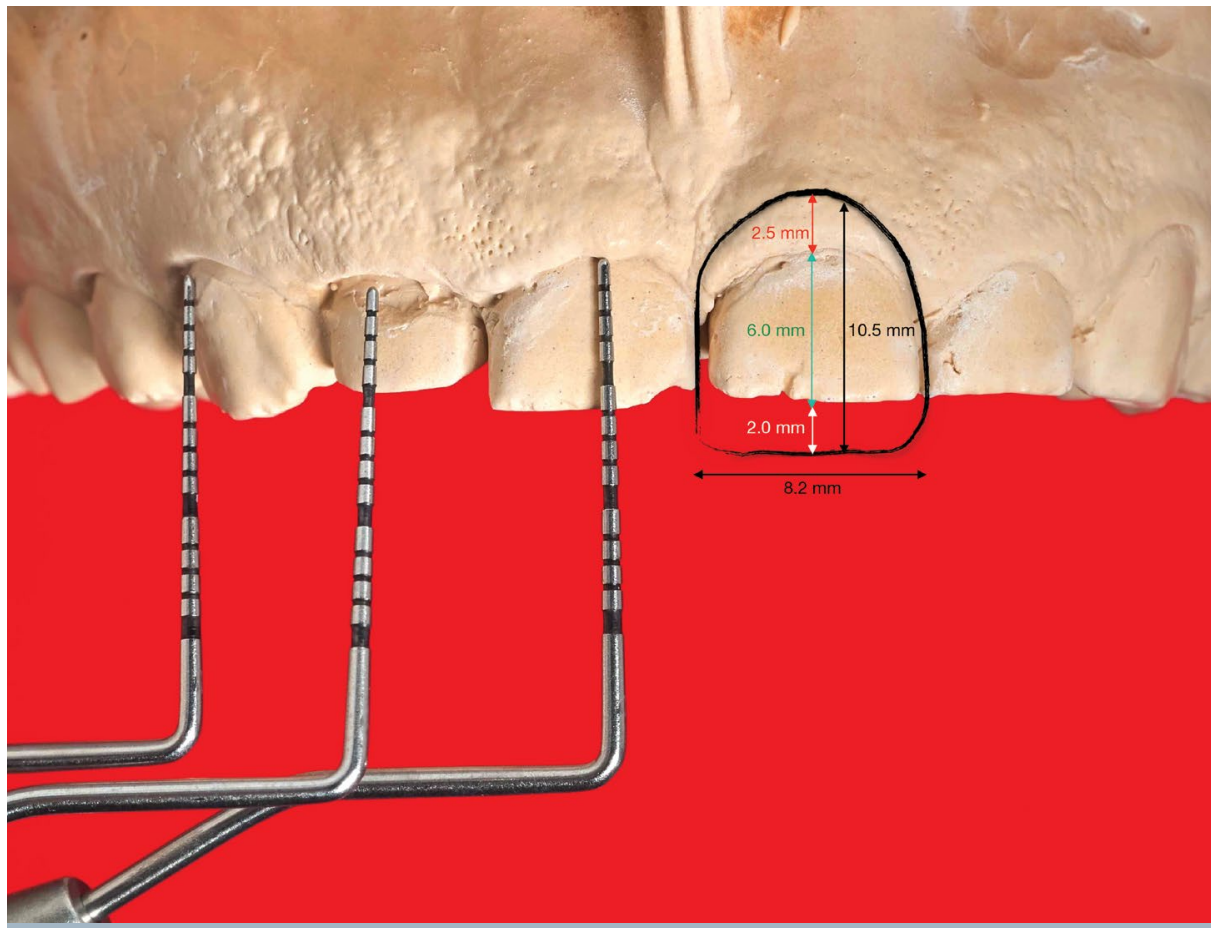

Fig. 12 The width/length $(w / I)$ ratio of the maxillary left central incisor is $0.78(8.2 / 10.5=0.78)$

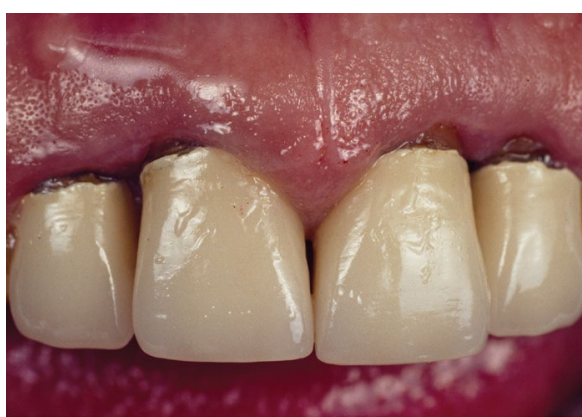

Fig. 9 Cervical black lines, and interproximal black triangles, around defective metalceramic prostheses due to visibility of metal substructure and gingival recession

dentistry is highly subjective, it should not be undertaken in a silos manner. Instead, a meticulous and systematic approach with multidisciplinary collaboration is required so that nothing is overlooked and all factors taken into consideration. Another issue to overcome with elective cosmetic procedures is resolving the ethical patient autonomy versus salutogenesis dilemma. The aims of treatment are minimally invasive, limited to as few teeth as possible for achieving the aesthetic objectives. Also, over-treatment should be avoided for seeking spurious 'ideals' that may be unattainable and detrimental in the long run. Finally, patient involvement cannot be over stressed, and approval is necessary at each stage before progressing to subsequent phases of treatment. It is far better to turn back halfway than be confronted with a culde-sac at the terminus.

\section{The seven deadly sins}

The following discussion highlights seven deadly sins of aesthetic dentistry that are culprits of a fake smile, how to spot them, and suggests guidelines for avoiding these pitfalls for creating inconspicuous and natural looking restorations.

\section{First sin - Cervical black lines}

During a relaxed smile, individuals with a high lip line reveal the cervical margins of their maxillary teeth, and if present, 'black lines' around artificial prostheses become blatantly visible (Fig. 9). These unsightly black lines are attributed to numerous causes, including defective margins, exposure of the metallic substructure of metal-ceramic crowns, shine through of underlying tooth discolouration via thin gingival margins (thin periodontal biotypes), gingival recession and shadowing by a pontic overlapping a deficient alveolar ridge. Elimination depends on the cause of the black lines.

If the cause is visibility of a metallic substructure, the latter can be concealed either 

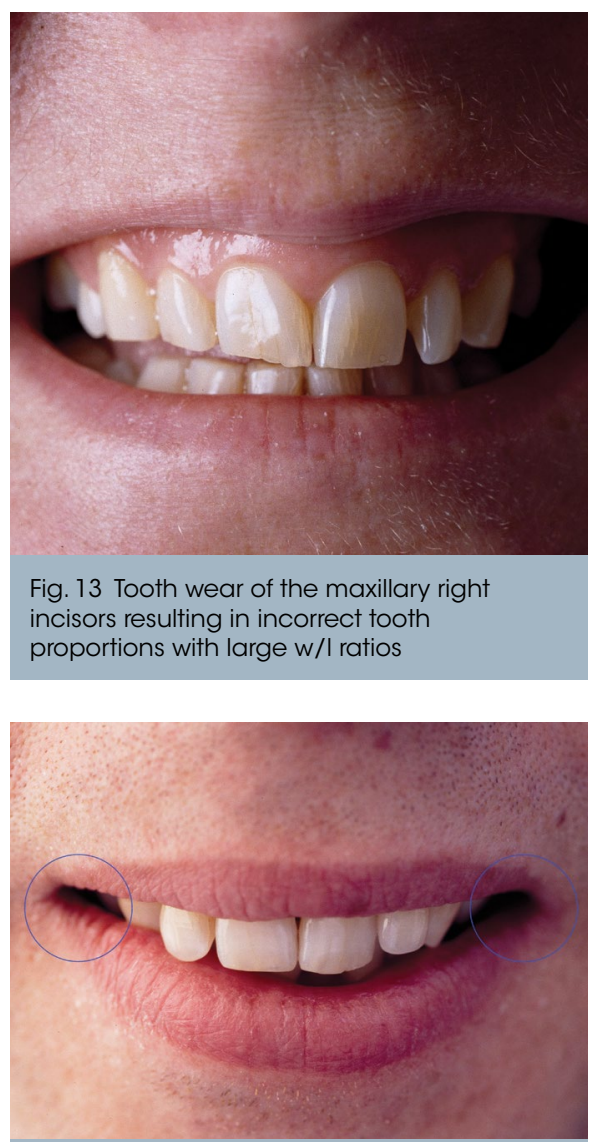

Fig. 15 Maintaining bilateral negative spaces is essential for pleasing aesthetics (as shown in image), and preventing excessive tooth display or a 'toothy' smile

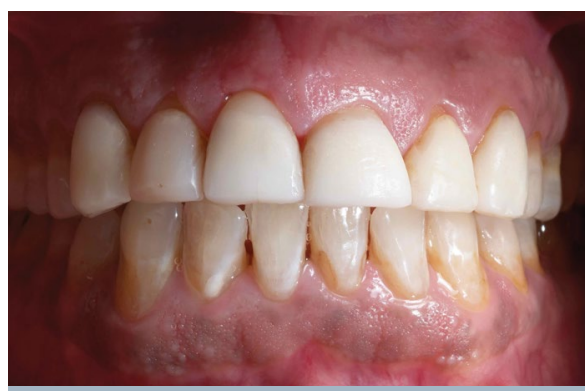

Fig. 17 Lack of incisal embrasures and dominance of the maxillary centrals of these composite fillings on the maxillary incisors and canines create monotony

by placing the crown margins subgingivally, or if occlusion permits, using an all-ceramic restoration with superior light transmission properties. Recession, on the other hand, is much more difficult to resolve. Often, when crowns are cemented the cervical margins appear to be subgingival. However, if the biologic width was inadvertently violated during crown preparation or other clinical procedures (gingival retraction, iatrogenic insult by rotary instrumentation, impression making or traumatic luting techniques), upon healing the resultant apical location of the free gingival margin (FGM) reveals the restoration/tooth interface, which is

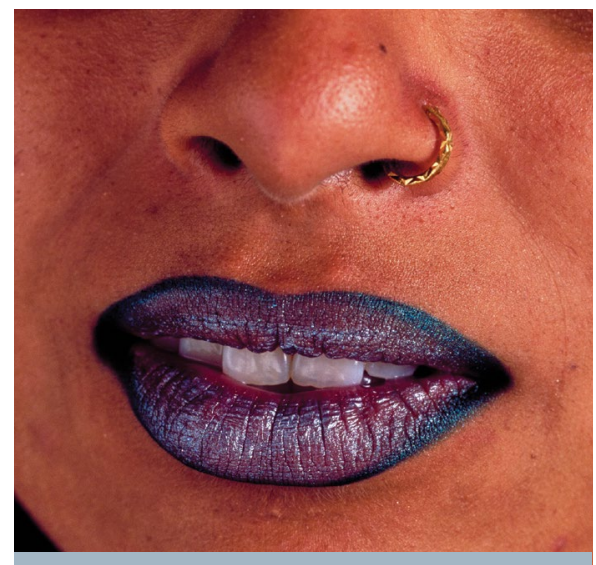

Fig. 14 A youthful and feminine smile is characterised by a greater degree of maxillary tooth display at the 'rest' position

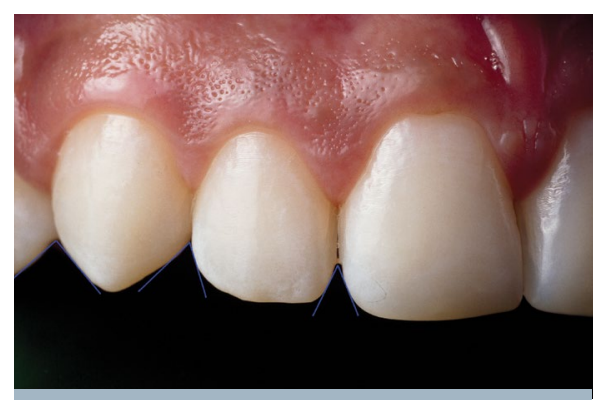

Fig. 16 In a natural dentition, the incisal embrasure angles increase anterior-posteriorly

detrimental to aesthetic appraisal. Differential diagnosis of gingival recession requires consideration of periodontitis or toothbrush abrasion, which leads to similar aesthetic problems, but necessitates a different approach for resolution. Methods to resolve gingival recession include concealing restorative margins by locating them subgingivally, or periodontal plastic surgery using gingival grafts for root coverage.

A particularly difficult scenario is recession around a discoloured non-vital or root filled tooth. In these cases, the options are internal non-vital bleaching, using a highly opaque luting agent or choosing a definitive restoration capable of obscuring the intrinsic discolouration. The optical and masking properties of porcelains vary enormously, and if translucent ceramics are chosen, the underlying tooth discolouration may not be adequately masked. Other options are either a metal-ceramic unit, or an all-ceramic restoration incorporating an optically dense core such as alumina or zirconia.

Lastly, shadowing by a pontic due to resorbed bone necessitates ridge augmentation by guided bone regeneration and/or soft tissue grafts. However, surgical intervention is protracted and costly, but nevertheless should be offered, even though the patient may reject it and opt for an aesthetic compromise.
Second sin - Colour mismatch An artificial crown which fails to blend with adjacent or antagonist teeth can be detected with relative ease (Fig. 10). Colour is a complex subject, which is often perplexing for both the clinician and ceramicist. Shade taking is accomplished by visual, and instrumental (digital) assessment or a combination of both. The visual method is chromatic differentiation by comparison using custom made or proprietary shade guides (eg VITA classical or VITA 3D-Master) with appropriate illumination at different colour temperatures (eg daylight - 6500K and incandescent light - 3000K) for avoiding metamerism, and correct colour rendering index (CRI) for preventing colour distortions. The instrumental option yields numerical absolute colour readouts without making a comparison to a reference using digital photographs, colour measuring devices (eg ShadeEye-NCC or Easyshade V) or intra-oral scanners (eg 3-Shape), used for taking digital impressions, that have in-built software tool function for shade evaluation corresponding to a variety of shade guides (Fig. 11). Since colour evaluation is idiosyncratic, it is worthwhile involving both the patient and ceramicist during the shade taking process, thereby avoiding disappointment at the delivery stage of the restoration. Another factor contributing to shade mismatch is characterisations. Even though the basic colour (hue, value, chroma) may be satisfactory, if nuances such as incisal and interproximal translucencies, opalescence, fluorescence or surface texture and lustre are incorrect, the effect is similar to a colour mismatch, resulting in conspicuous restorations.

Third sin - Incorrect tooth proportions Maxillary anterior teeth have distinct proportions and specific relationship between the incisors and canines for ensuring harmony and balance. However, contralateral symmetry should be avoided as it conveys perfection and a sense of artificiality. Nature is beautiful, not perfect. The method for determining the correct tooth size is the mesio-distal width divided by the incisogingival length, termed the width/length (w/l) ratio. The $\mathrm{w} / \mathrm{l}$ of the incisors and canines ranges from 0.6 to 1.0, with an accepted norm of 0.78 . Translated into actual dimensions, a $0.78 \mathrm{w} / \mathrm{l}$ ratio creates a central incisor which is $10.5 \mathrm{~mm}$ long and $8.2 \mathrm{~mm}$ wide $(8.2 / 10.5=0.78)$ - Fig.12. The colloquially used phase 'long in the tooth' describes an aged person, usually associated with periodontal disease and subsequent gingival recession creating the appearance of long teeth with smaller w/l ratios. Conversely, excessive incisal tooth wear has the opposite effect of creating short, wide teeth with large w/l ratios that are equally unappealing (Fig. 13). 


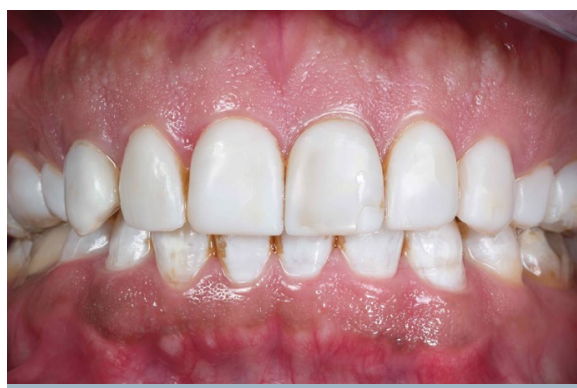

Fig. 18 Providing monochromatic restorations conveys artificiality, rarely observed in the natural dentition
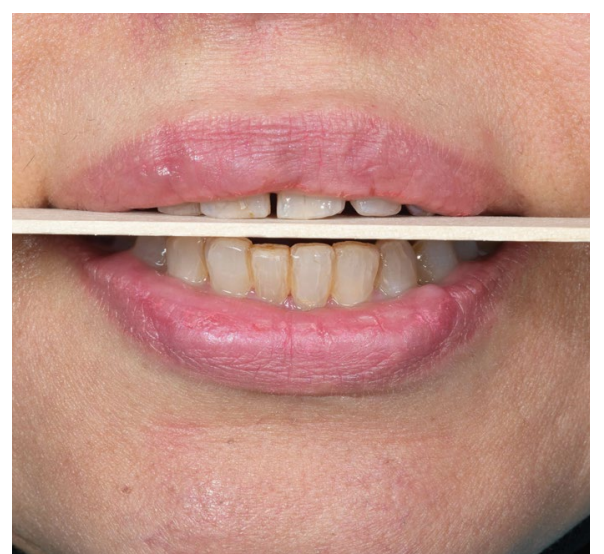

Fig. 21 Assessing the incisal plane inclination by biting on a wooden spatula aligned to the horizon
Fig. 19 A maxillary arch restored with veneers, crowns and bridges showing the incisal plane coincident with the curvature of the lower lip during smiling

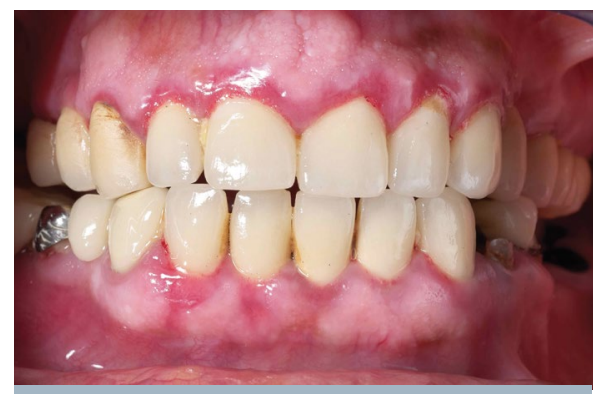

Fig. 22 Inflammation of the free gingival margin (FGM) around the crowns on the anterior maxillary and mandibular teeth, compromising pink aesthetics
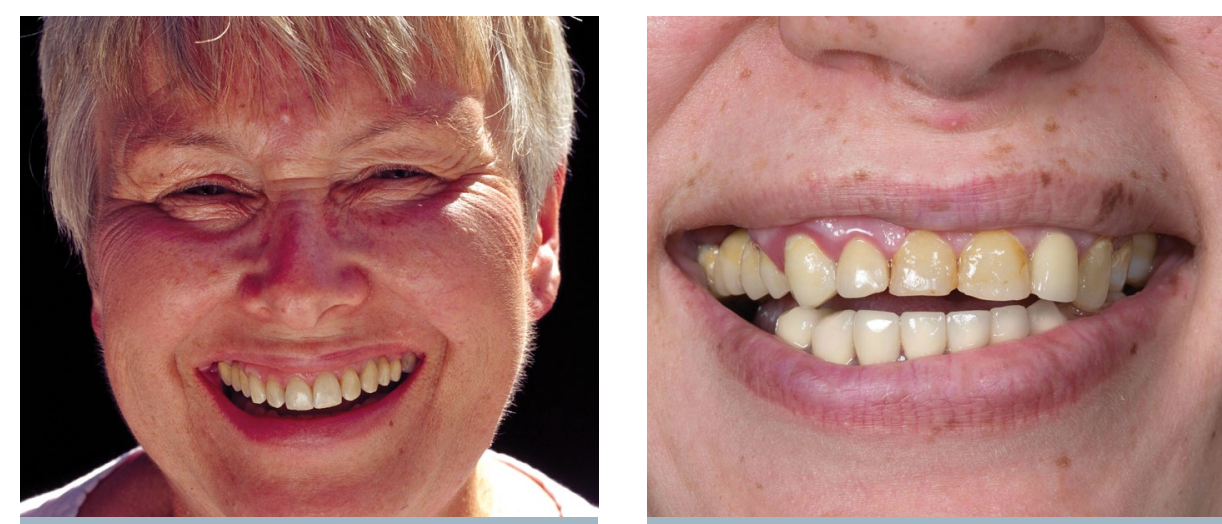

Fig. 20 Defective maxillary restorations with a convex incisal/occlusal plane not parallel with the concave curvature of the lower lip

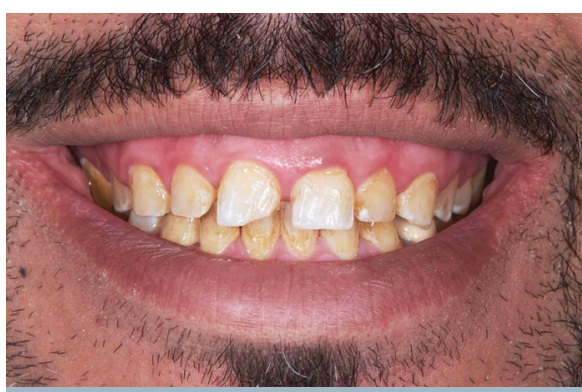

Fig. 23 Aesthetic crown lengthening: preoperative status showing greater than $3 \mathrm{~mm}$ maxillary gingival exposure during a relaxed smile

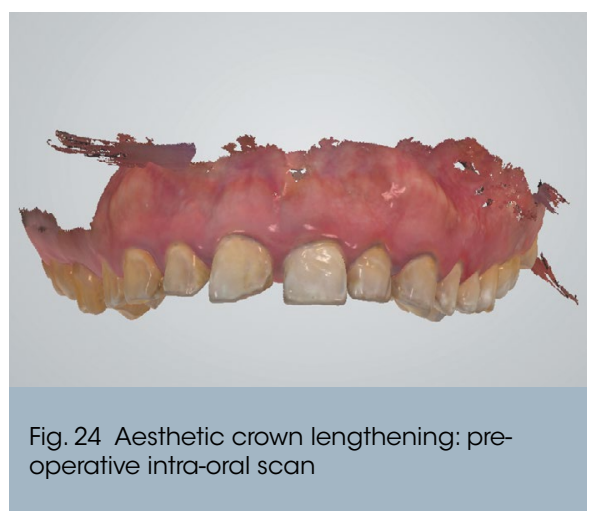

smile may appear full or 'toothy' due to several excessive bulbous restorations that mistakenly 'close' the lateral negative spaces (buccal corridors) bordering the buccal aspect of the teeth and inner aspects of the lips (Fig. 15).

Bulbosity can be isolated to a single tooth or encompass numerous teeth. A 'goofy' appearance is attributed to extreme bulbosity, with protrusive and prominent anterior teeth. Factors contributing to this anomaly include facial or buccal inclination of teeth often observed in an Angle's Class II occlusion, incorrect angulation of artificial units or bulbous artificial restorations when insufficient tooth reduction results in inadequate space for the prosthetic materials (substructure and/ or veneering porcelain), with resulting overcontoured units. In other circumstances, a
Fifth sin - Monotony

The two constituents of monotony in dental aesthetics are tooth-to-tooth relationship, and tooth shade. An ideal tooth-to-tooth relationship incorporates incisal embrasures with increasing angles traversing anteriorposteriorly (Fig. 16). If incisal embrasures are flat, due to tooth surface loss (tooth wear) or incorrect shape/form of dental prostheses, the effect is uniformity and monotony. In addition, the maxillary central incisors should smile is correlated to the degree of maxillary gingival exposure and the relationship of the 


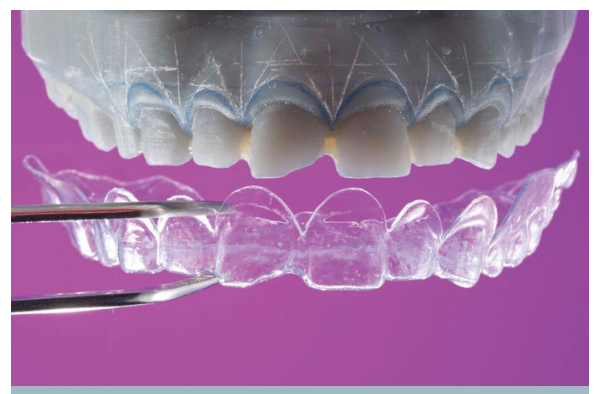

Fig. 25 Aesthetic crown lengthening: markings for crown lengthening surgery on 3D printed maxillary arch and a transparent surgical stent for guiding initial gingivectomy

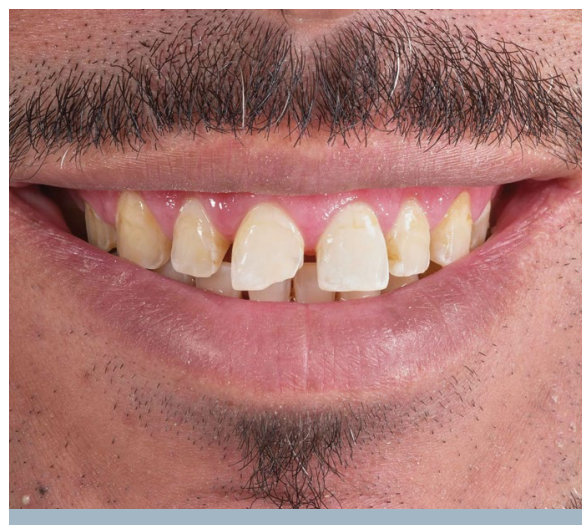

Fig. 27 Aesthetic crown lengthening: resolution of pink aesthetics (after three months' healing) before proceeding to rectify white aesthetics

dominate the dental composition, and be wider and longer than the adjacent laterals (Fig. 17).

Another point of contention is fabricating restorations that conform to the Golden proportion to justify 'extreme smile makeovers'. First, only $17 \%$ of the population have natural teeth conforming to the Golden proportion. Second, humans are not clones, but individuals with unique facial and dental features. Third, tooth preparation involving destruction of virgin enamel and dentine solely for fabricating prostheses that conform to the Golden proportion is at best spurious, and at worst unethical. Instant gratification cannot justify possible long-term spiralling dismay such as post-operative sensitivity, dislodgement of restorations, endodontic or periodontal complications, or extractions followed by implant placement. It is worth remembering the adage 'short term gain, long term pain' before embarking on this type of questionable therapy.

Similar to distinct form, teeth also exhibit distinct colour. Each tooth in a given arch has a unique shade, and it is rare for all teeth to be of an identical colour. For example, moving from the maxillary incisors to canines, the shade changes may be $\mathrm{A} 2$ for the centrals, A3 for the laterals and A3.5 for

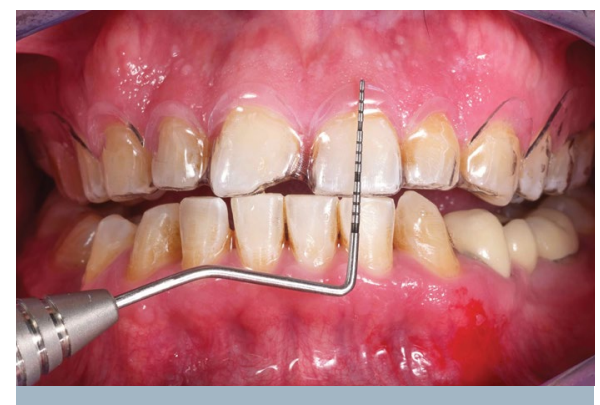

Fig. 26 Aesthetic crown lengthening: intraoral location of transparent surgical stent for guiding initial gingivectomy

the canines. Furthermore, each tooth has specific characterisations such as deep chroma cervical regions, incisal and interproximal translucent areas, as well as mamelons, cracks and distinctive surface roughness. Therefore, providing monochromatic restorations that are characterless convey artificiality - rarely observed in the natural dentition - and is contradicted for truly aesthetic restorations. Monotony is yet another penchant for 'extreme smile makeovers' by creating a uniform shade for a 'Barbie doll' unnatural façade (Fig. 18). While this type of 'fake it to make it' cover-up may be satisfactory for ephemeral cosmetic make-up, an irreversible dental makeover cannot be wiped away the following day with a cleansing cloth!

\section{Sixth sin - Slanted smile}

Psychologically, a concave shape signifies welcome and receptiveness, while a convex form conveys aggressiveness and belligerence. For example, holding out your hand for a handshake is a welcoming gesture whereas a hand forming a concave shaped fist implies confrontation and wrath. Similarly, a smile should be concave, with the incisal or occlusal plane of the maxillary teeth parallel to the concave curvature of the lower lip (Fig. 19). This incisal inclination may be absent due to unilateral or isolated tooth wear, erratic eruption patterns, periodontal disease, tooth malpositions, slanted maxilla or defective restorations with incorrect maxillary occlusal/ incisal plane (Fig. 20). The methods to assess correct angulations of the incisal plane are using a face bow, or taking a photograph with the patient biting on a wooden spatula that is aligned to the horizon (Fig. 21). The two key points to note are that the incisal plane should coincide with the interpupillary line, and the curvature of the lower lip during a relaxed smile.

\section{Seventh sin - Gingival abnormalities}

Surreptitiously, a low maxillary lip line conceals a multitude of sins, while a high lip line exposes them. In many instances, all the above sins may be absent, black cervical lines, colour mismatch, incorrect tooth proportions, bulbosity, monotony and slanted smile, yet something is still missing. If this is the case, the cause is probably gingival abnormalities or lack of pink and/or facial aesthetics. Pink aesthetics encompasses a myriad of soft tissue features. When attending a lecture on dental aesthetics, an easy way to pick out an artificial restoration is to move your eyes apically and view the soft tissues. An obvious give away is inflammation of the free gingival margins (FGM) - Figure 22 - or presence of open gingival embrasures creating interproximal 'black triangles' between artificial restorations. The reasons for the latter could be defective restorative margins, violation of the biologic width, incorrect emergence profile or simply poor oral hygiene. Whatever the reason, a swollen FGM is not only detrimental to pink aesthetics, but also negatively impacts on the longevity of a restoration.

Another less palpable aberration is the peaks or zeniths of the FGM around the incisors and canines, classified as the gingival aesthetic line or GAL. There are four classes of GAL, eg, in GAL Class I the zeniths of the FGM of the maxillary central incisor and canine lie on the same tangent, while the lateral incisor is 1 to $2 \mathrm{~mm}$ below this imaginary line. Methods to rectify erratic gingival zeniths include perioplastic surgery (aesthetic crown lengthening, root coverage), and/or orthodontic movement of the dentogingival complex either in an apical or coronal location.

Finally, a 'gummy smile' is associated with excessive maxillary gingival display apical to the cervical margins of the maxillary teeth. During a relaxed smile, up to $3 \mathrm{~mm}$ of gingival exposure apical to the FGM is acceptable. However, beyond this arbitrary limit, pink aesthetics are severely compromised. Correction of excessive gingival display is cause related, ie skeletal, muscular or dentogingival abnormalities including elongated maxillae, short and/or hypertonic maxillary lips, dentoalveolar compensation, Angle's Class II (ii) or Class III occlusions, altered passive eruption (APE) or a combination of these factors. Some procedures for rectifying these conditions are orthognathic surgery, orthodontic movement, and aesthetic crown lengthening (gingivectomy with or without ostectomy and osteoplasty) - Figures 23, 24, 25 , 26, 27. Alternately, surgical lip repositioning, and non-surgical facial aesthetics (NSFA) with Botox injections are ideal for fine tuning a smile and resolving both pink and facial aesthetics. 


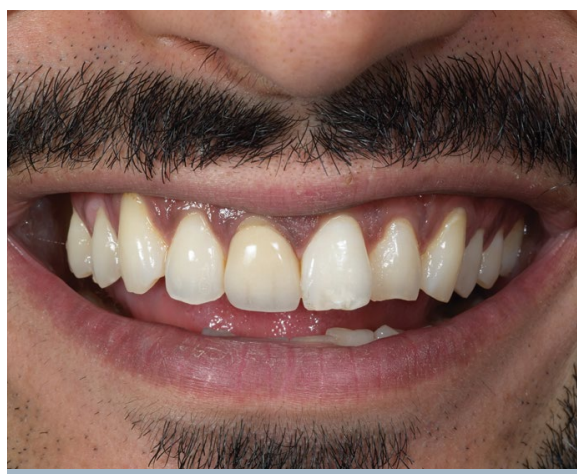

Fig. 28 Dento-facial pre-operative view of defective crown on maxillary right central incisor

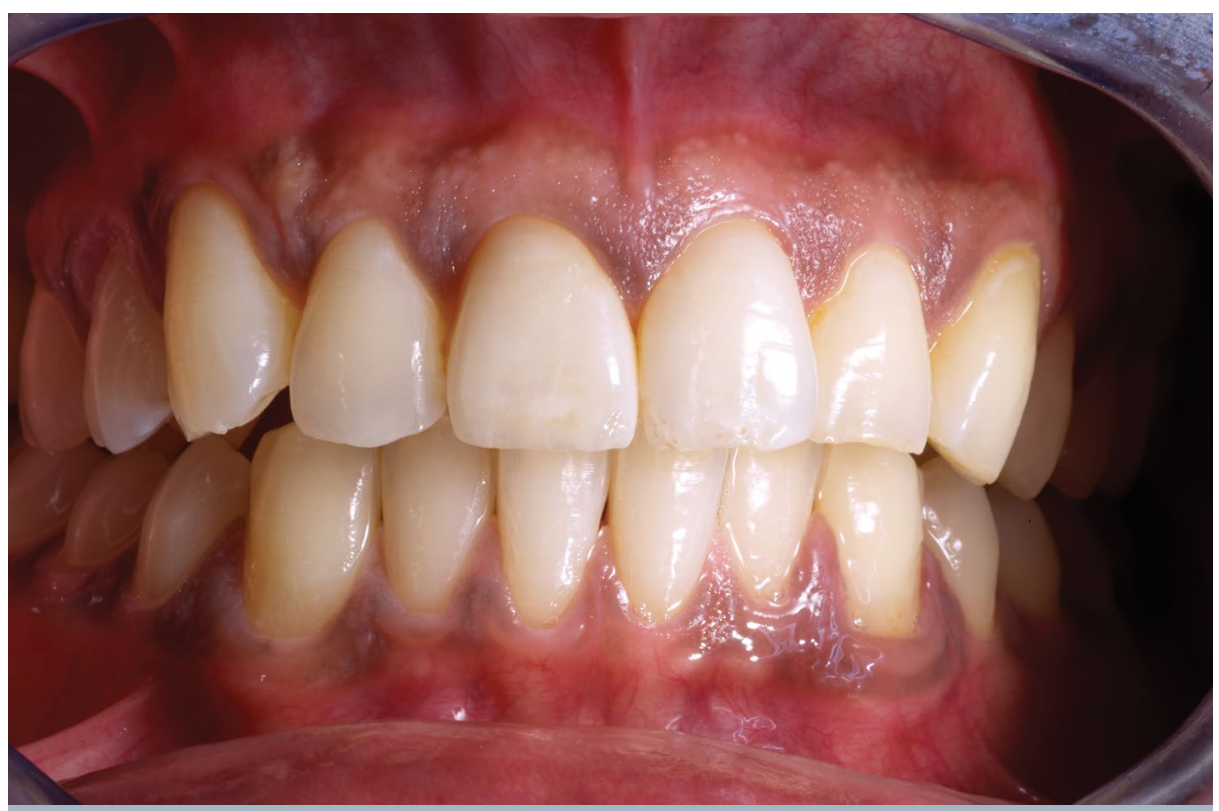

Fig. 31 Post-operative replacement with an all-ceramic lithium disilicate (e-max) crown on maxillary right central incisor

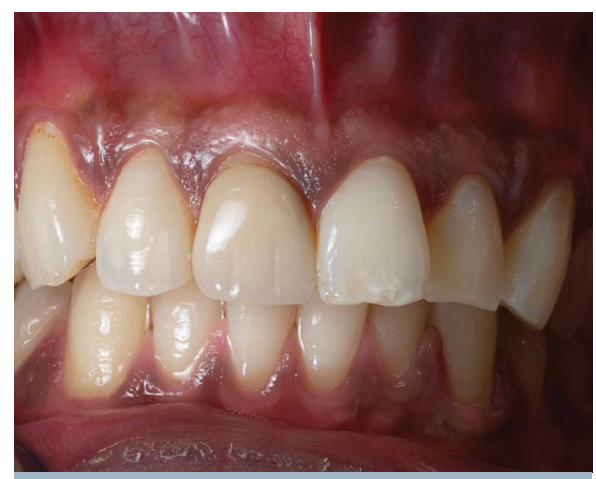

Fig. 29 Dental pre-operative view of defective crown on maxillary right central incisor

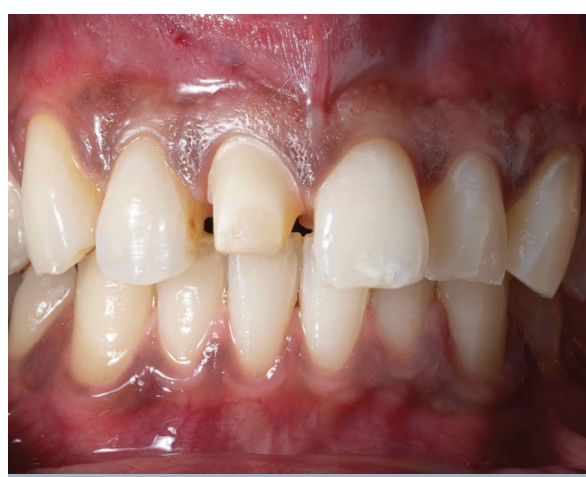

Fig. 30 Removal of defective crown and refined tooth preparation

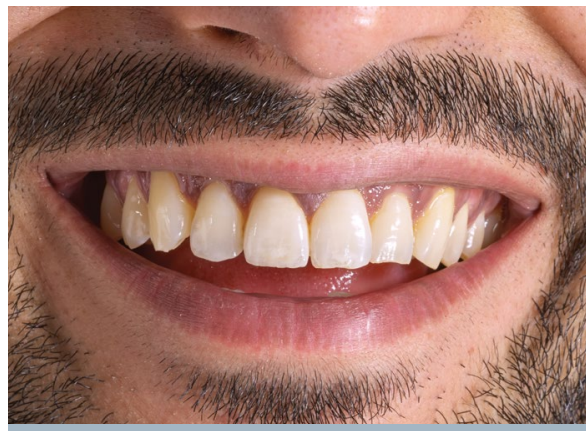

Fig. 32 Dento-facial post-operative view of replacement crown on maxillary right central incisor that impeccably integrates with the surrounding dentition and soft tissues, restoring both white and pink aesthetics, respectively

dental restorations. Blackwell Munksgaard, 2006.

4. Trushkowsky R. Esthetic oral rehabilitation with veneers. Springer, 2020.

5. Al-Harbi F, Ahmad I. A guide to minimally invasive crown lengthening and tooth preparation for rehabilitating pink and white aesthetics. Br Dent J 2018; 224: 228-234.

6. Ahmad I, Al-Harbi F. 3D printing in dentistry 2019/2020. Quintessence Publishing Co., 2019.

7. Ahmad I. Essentials of dental photography. Wiley-Blackwell, 2019.

8. Chu S J, Devigus A, Paravina R, Mieleszko A. Fundamentals of color: shade matching and communication in esthetic dentistry, 2nd edition. Quintessence Publishing Co., 2011.

9. Romano R. Art of the smile. Quintessence Publishing Co., 2005.

10. Ahmad I. Prosthodontics at a glance, 2nd edition. Wiley-Blackwell, 2021.

\section{Further reading}

1. Ahmad I. Risk management in clinical practice. Part 5. Ethical considerations for dental enhancement procedures. Br Dent $J$ 2010; 209: 207-214.

2. Ahmad I. A clinical guide to anterior dental aesthetics. London: BDJ Books, 2005.

3. Ahmad I. Protocols for predictable aesthetic
An earlier version of this article was published in Vital in 2006. With thanks to the author for updating the text for 2021 and providing new images.

https://doi.org/10.1038/s41407-021-0619-7 